\title{
FISSION OF ACTINIDES AND SUPERHEAVY NUCLEI: COVARIANT DENSITY FUNCTIONAL PERSPECTIVE
}

\author{
A. V. AFANASJEV* \\ Department Physics and Astronomy, Mississippi State University, \\ Mississippi State, MS 39762, USA \\ *E-mail: afansjev@erc.msstate.edu
}

\begin{abstract}
The current status of the application of covariant density functional theory to the description of fission barriers in actinides and superheavy nuclei is reviewed. The achievements and open problems are discussed.

Keywords: fission barriers, covariant density functional theory, actinides, superheavy nuclei
\end{abstract}

\section{Introduction}

A study of the fission barrier heights $B_{f}$ of nuclei is motivated by the importance of this quantity for several physical phenomena. For example, the $r$-process of stellar nucleosynthesis depends (among other quantities such as masses and $\beta$-decay rates) on the fission barriers of very neutronrich nuclei ${ }^{1,2}$. The population and survival of hyperdeformed states at high spin also depends on the fission barriers ${ }^{3,4}$. In addition, the physics of fission barriers is intimately connected with on-going search for new superheavy elements (SHE). The probability for the formation of a SHE in a heavyion-fusion reaction is directly connected to the height of its fission barrier ${ }^{5}$; the large sensitivity of the cross section $\sigma$ for the synthesis of the fissioning nuclei on the barrier height $B_{f}$ also stresses a need for accurate calculations of this value. The survival of the actinides and SHE against spontaneous fission depends on the fission barrier which is a measure of the stability of a nucleus reflected in the spontaneous fission lifetimes of these nuclei ${ }^{7}$.

The recent progress in the description of fission barriers within covariant density functional theory $\left(\mathrm{CDFT}^{6}\right)$ is briefly reviewed in the current manuscript. The comparison with the results of other model calculations is presented. Open problems in the study of fission barriers are also discussed. 


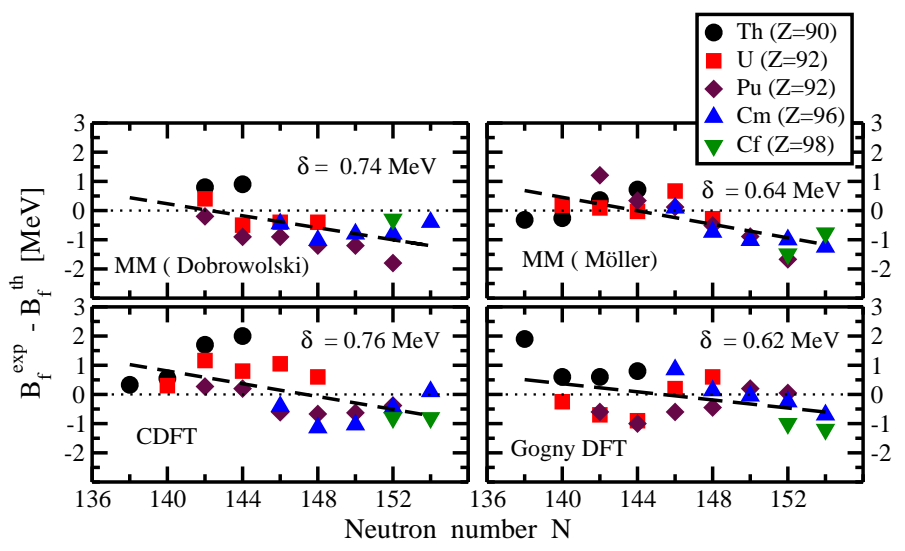

Fig. 1. The difference between experimental and calculated heights of inner fission barriers as a function of neutron number $N$. The results of the calculations are compared to estimated fission barrier heights given in the RIPL-3 database ${ }^{8}$, which is used for this purpose in the absolute majority of theoretical studies on fission barriers in actinides. The results of the calculations within microscopic+macroscopic method ('MM(Dobrowolski)' 9 and 'MM(Möller)' 10), covariant density functional theory ('CDFT' 11) and density functional theory based on the finite range Gogny force ('Gogny DFT' 12) are shown. Thick dashed lines are used to show the average trend of the deviations between theory and experiment as a function of neutron number. The average deviation per barrier $\delta$ [in $\mathrm{MeV}]$ is defined as $\delta=\sum_{i=1}^{N}\left|B_{f}^{i}(t h)-B_{f}^{i}(\exp )\right| / N$, where $N$ is the number of the barriers with known experimental heights, and $B_{f}^{i}(t h)\left(B_{f}^{i}(\exp )\right)$ are calculated (experimental) heights of the barriers. Long-dashed lines represent the trend of the deviations between theory and experiment as a function of neutron number. They are obtained via linear regression based on a least square fit. From Ref. ${ }^{13}$.

\section{Current status of the investigation of fission barriers in actinides}

The progress in the development of computer codes and the availability of powerful computers has allowed to study in a systematic way the effects of triaxiality on the fission barriers leading to their realistic description. Within the CDFT framework, the inner fission barriers with triaxiality included have been studied for the first time in Ref. ${ }^{11}$ using triaxial RMF+BCS approach and the NL3* parametrization ${ }^{14}$. Two years later, similar studies of fission barriers in actinides have been performed in Refs. ${ }^{15,16}$ using the RMF+BCS framework with the PC-PK1 and DD-PC1 parametrizations of the RMF Lagrangian. The accuracy of the description of the heights of inner fission barriers in these calculations is comparable with the one obtained in Ref. ${ }^{11}$. The calculations of Refs. ${ }^{15,16}$ also include the results for outer fission barriers where the effects of octupole deforma- 
tion (and triaxiality [Ref. $\left.{ }^{15}\right]$ ) are taken into account. They agree reasonably well with experimental data.

As compared with axially symmetric calculations, the inclusion of triaxiality has drastically improved the accuracy of the description of inner fission barriers in all model calculations (see Ref. ${ }^{13}$ and references quoted therein). Fig. $1^{\mathrm{a}}$ shows that the state-of-the-art calculations within different theoretical frameworks (including CDFT) are characterized by comparable accuracy (the $\delta$-values) of the description of inner fission barriers. Note that this comparison covers only the results of systematic triaxial calculations of even-even Th, U, Pu, Cm and Cf nuclei. Recent calculations within Skyrme DFT are also characterized by similar accuracy of the description of fission barriers ${ }^{17,18}$.

Minor differences between the approaches in the obtained average deviations per barrier (Fig. 1) are not important considering the considerable uncertainties in the extraction of inner fission barrier heights from experimental data (see discussion in Ref. ${ }^{19}$ ). However, the similarity of the average trends of these deviations (shown by thick dashed lines in Fig. 1) as a function of neutron and proton numbers is more important considering the differences in underlying mean fields and in the treatment of pairing correlations. At present, no clear explanation for these trends is obtained 13,19 .

The investigations of Refs. ${ }^{11,15,16}$ clearly indicate that good description of fission barriers in actinides in the CDFT framework is obtained with the parametrizations fitted with no information on fission barriers. On the contrary, successful description of fission barriers in actinides within nonrelativistic DFT is based on the parametrizations which explicitly use either fission barrier heights (SkM* in Skyrme DFT and D1S in Gogny DFT) or fission isomer excitation energies (UNEDF1 in Skyrme DFT); the latter being strongly correlated with inner fission barrier height.

\section{Fission barriers in superheavy nuclei.}

Fig. 2 shows how the models which have been bench-marked in a systematic way in the actinides (see Fig. 1) extrapolate to the region of superheavy nuclei. Note that the results of the MM calculations of Ref. ${ }^{20}$ labeled as 'MM(Kowal)' are not shown on Fig. 1. However, they describe inner fission barriers of actinides very accurately. One can see that the model predictions vary wildly; the difference in inner fission barrier heights between different

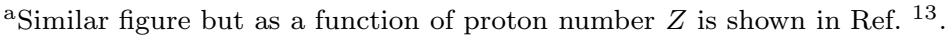




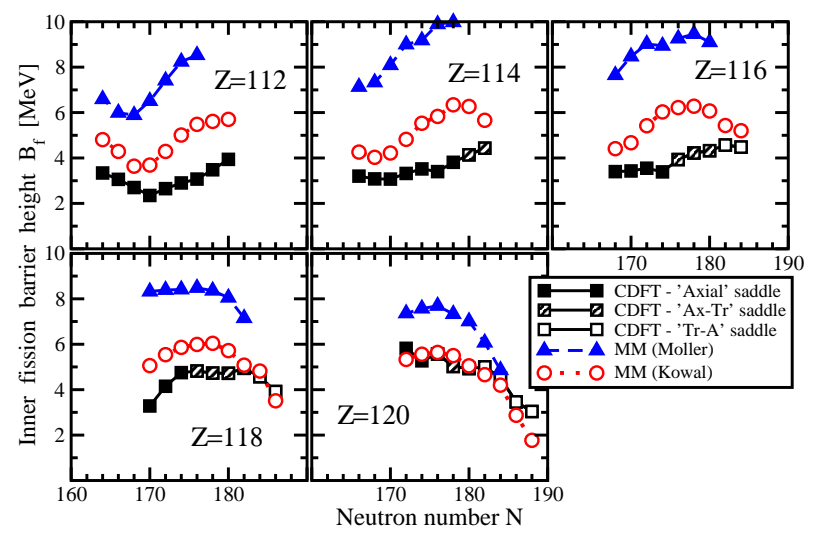

Fig. 2. Inner fission barrier heights $B_{f}$ as a function of neutron number $N$. The results of the MM calculations are taken from Ref. ${ }^{10}$ (labeled as 'MM (Möller)') and Ref. ${ }^{20}$ (labeled as 'MM (Kowal)'). The position of inner fission barrier saddle in deformation space varies as a function of particle number. The labeling of Ref. ${ }^{13}$ is used is order to indicate whether the saddle is axial (labeled as 'Axial'), has small $\left(\gamma \sim 10^{\circ}\right.$, labeled as 'Ax-Tr') or large $\left(\gamma \sim 25^{\circ}\right.$, labeled as 'Tr-A') $\gamma$-deformations in the RMF+BCS calculations.

models reaches $6 \mathrm{MeV}$ in some nuclei. This is despite the fact that these models describe the inner fission barriers in actinides with comparable level of accuracy. The more surprising fact is that the prediction of two MM models differ so substantially; in reality the 'MM (Kowal)' model predictions are closer to the CDFT ones than to the 'MM (Möller)' predictions.

\section{Some open questions}

The results in the SHE region raise a number of questions which require further investigation. Among these, for example, are

- A. How the pairing evolves with deformation? How well the pairing strength is defined for SHE?

- B. How the accuracy of the description of the energies of the singleparticle states and its evolution with deformation affects the results of the calculations?

I will concentrate on question A. Table IV in Ref. ${ }^{13}$ shows the large variety of pairing prescriptions used in the calculations of fission barriers during last decade. The question definitely emerges how well they describe the evolution of pairing with deformation and extrapolate towards SHE. These features are important for a quantitative description of fission barrier 


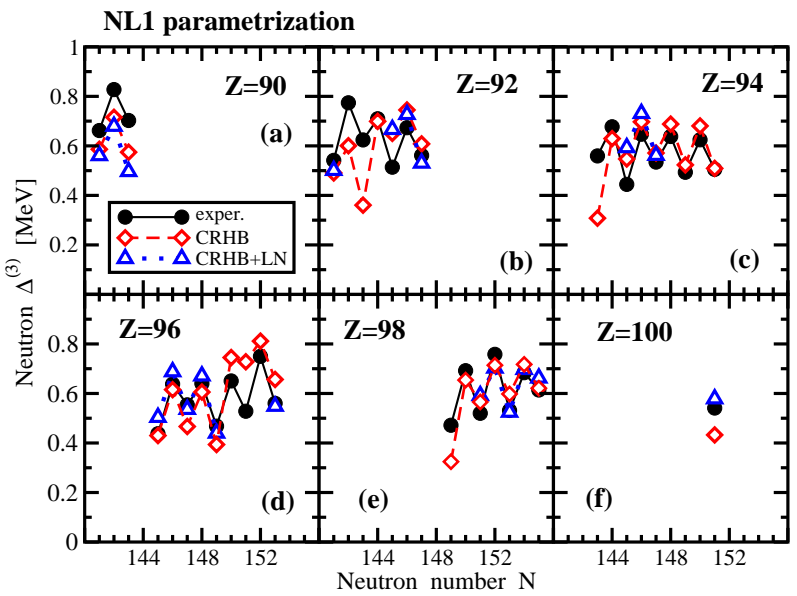

Fig. 3. Experimental and calculated neutron three-point indicators $\Delta_{\nu}^{(3)}(N)$ as a function of neutron number $N$. The results of the CRHB and CRHB+LN calculations with the NL1 parametrization are shown.

heights which according to Ref. ${ }^{23}$ sensitively depend on pairing properties. Although some attempts were made in 70ies to extract the information on pairing properties at fission saddles of actinides ${ }^{24}$, they did not lead to reliable estimates. It is even more difficult to get information on pairing properties of SHE due to the lack of relevant experimental data. As a consequence, as accurate as possible description of pairing properties in actinides and light SHE should be considered as a necessary condition for further extrapolation to heavier SHE.

In order to address these questions, a comprehensive study of pairing and rotational properties of actinides is performed in Ref. ${ }^{25}$ in the cranked relativistic Hartree-Bogoliubov (CRHB) framework. In this approach, the Brink-Booker part of phenomenological non-relativistic Gogny-type finite range interaction

$$
V^{p p}(1,2)=f \sum_{i=1,2} e^{-\left[\left(\boldsymbol{r}_{1}-\boldsymbol{r}_{2}\right) / \mu_{i}\right]^{2}}\left(W_{i}+B_{i} P^{\sigma}-H_{i} P^{\tau}-M_{i} P^{\sigma} P^{\tau}\right)
$$

is used in pairing channel. In addition, an approximate particle number projection by means of Lipkin-Nogami (LN) method is employed. The analysis indicates that the pairing strength has to be decreased as compared with original D1S Gogny force; the scaling factor $f$ is equal to 0.899 and 0.9147 for the NL3* and NL1 parametrizations, respectively. Only with these scaling factors, the moments of inertia and odd-even mass staggerings (the $\Delta^{(3)}$ indicators) can be well described in the normal-deformed (ND) minimum 


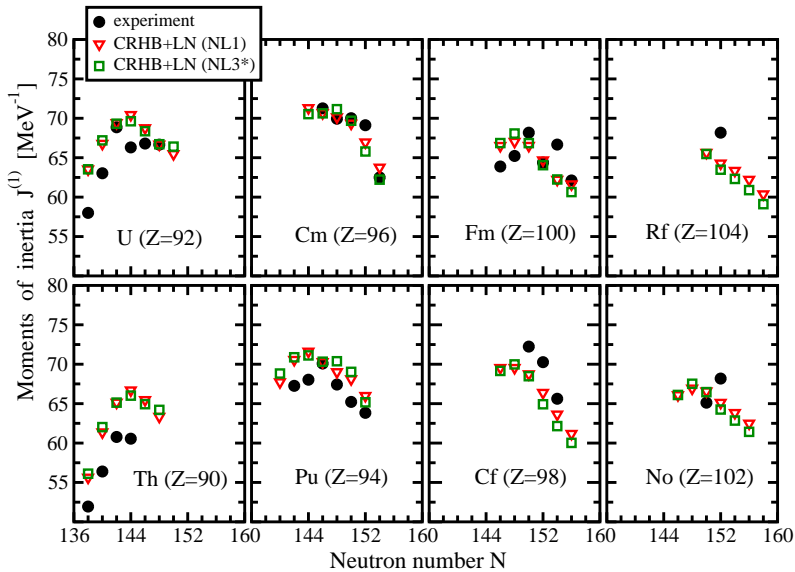

Fig. 4. Calculated and experimental moments of inertia at low spin.

(Figs. 3 and 4).

Table 1. Experimental and theoretical charge quadrupole moments $Q$ of SD fission isomers. The results of the CRHB+LN calculations with the NL1 and NL3* parametrizations are presented. Experimental data for the $\mathrm{U}$ and $\mathrm{Pu}$ isotopes are taken from Ref. ${ }^{21}$, while the one for ${ }^{242} \mathrm{Am}$ from Ref. ${ }^{22}$.

\begin{tabular}{ccccccc}
\hline & ${ }^{236} \mathrm{U}$ & ${ }^{238} \mathrm{U}$ & ${ }^{236} \mathrm{Pu}$ & ${ }^{239} \mathrm{Pu}$ & ${ }^{240} \mathrm{Pu}$ & ${ }^{242} \mathrm{Am}$ \\
\hline$Q^{\text {exp }}(e \mathrm{~b})$ & $32 \pm 5$ & $29 \pm 3$ & $37 \pm 10$ & $36 \pm 4$ & & $35.5 \pm 1.0_{\text {st }} \pm 1.2_{\text {mod }}$ \\
$Q^{\mathrm{NL} 1}(\mathrm{eb})$ & 35.8 & 37.3 & 36.1 & & 38.2 & \\
$Q^{\mathrm{NL} 3 *}(\mathrm{eb})$ & 33.9 & 33.7 & 34.8 & & 34.9 & \\
\hline
\end{tabular}

Fission isomers provide only available tool to estimate the evolution of pairing with deformation in actinides. Such an estimate is available only through the study of rotational properties of ${ }^{236,238} \mathrm{U}$ and ${ }^{240} \mathrm{Pu}$ nuclei; these are only nuclei for which superdeformed (SD) rotational bands were experimentally measured. The calculated charge quadrupole moments $Q$ are compared with available experimental data in Table 1. The CRHB+LN(NL3*) results of the calculations come reasonably close to experiment. The $\mathrm{CRHB}+\mathrm{LN}(\mathrm{NL} 1)$ results are also not far away from experimental data but they substantially overestimate experimental $Q$ value in ${ }^{238} \mathrm{U}$.

The experimental kinematic moments of inertia are best described by the NL3* parametrization; the deviation from experiment does not exceed $3.4 \%$ (Fig. 5). The fact that the moments of inertia of rotational structures in two different minima (ND and SD) are accurately described with the 
same pairing strength strongly suggests that the evolution of pairing correlations with deformation is properly described in the $\mathrm{CRHB}+\mathrm{LN}\left(\mathrm{NL} 3^{*}\right)$ framework by the Gogny D1S pairing.

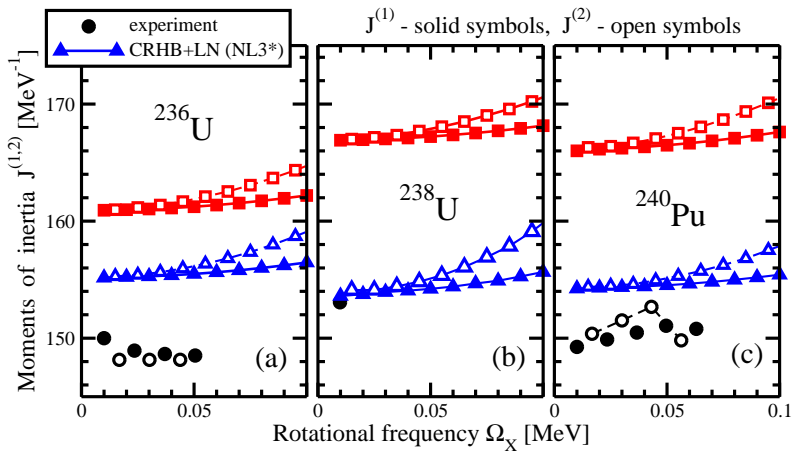

Fig. 5. Experimental and calculated kinematic $\left(J^{(1)}\right)$ and dynamic $\left(J^{(2)}\right)$ moments of inertia of SD rotational bands in ${ }^{236,238} \mathrm{U}$ and ${ }^{240} \mathrm{Pu}$. The notation of the lines and symbols is given in the figure.

However, this is not always the case since the CRHB+LN(NL1) calculations substantially overestimate the experimental moments of inertia in the SD minimum [for example, by $11.3 \%$ in ${ }^{240} \mathrm{Pu}$ ] (Fig. 5) while they reproduce the low-spin moments of inertia in the ND minimum with the same level of accuracy as the $\mathrm{CRHB}+\mathrm{LN}\left(\mathrm{NL}^{*}\right.$ ) calculations (see Fig. 4). It turns out that reasonable description of the moments of inertia at SD can be achieved in the $\mathrm{CRHB}+\mathrm{LN}(\mathrm{NL} 1)$ calculations only if scaling factor $f \approx 1.0$ is used. This clearly indicates that even the pairing force carefully fitted to experimental data at ND does not guarantee accurate description of pairing at SD (and as a consequence also at fission saddle).

The origin of such behavior is not completely clear but the difference between the $\mathrm{CRHB}+\mathrm{LN}\left(\mathrm{NL} 3^{*}\right)$ and $\mathrm{CRHB}+\mathrm{LN}\left(\mathrm{NL} 1^{*}\right)$ results for $J^{(1)}$ at $\mathrm{SD}$ may also partially originate from the differences in the single-particle structures at superdeformation obtained with the NL3* and NL1 parametrizations. The $Q$ values obtained in the $\mathrm{CRHB}+\mathrm{LN}(\mathrm{NL} 1)$ calculations are always higher than the ones for $\mathrm{CRHB}+\mathrm{LN}\left(\mathrm{NL} 3^{*}\right)$, which also may be a reason why the $\mathrm{CRHB}+\mathrm{LN}(\mathrm{NL} 1)$ calculations systematically overestimate kinematic moments of inertia at SD.

\section{Conclusions}

The review of the recent applications of covariant density functional theory to the description of fission barriers in actinides and superheavy nuclei is 
presented. Inner fission barriers of actinides are described with comparable accuracy in the MM and DFT (including CDFT) calculations. However, the predictions for fission barriers of SHE within different frameworks vary drastically indicating the need for better parametrizations of different channels of the models. In particular, a better pairing force and its better parametrization is needed. An effort to improve the Gogny pairing force in the RHB framework, the first results of which are presented here, is currently in progress.

\section{Acknowledgments}

This work has been supported by the U.S. Department of Energy under the grant DE-FG02-07ER41459. I would like to express my gratitude to P. Ring, H. Abusara and O. Abdurazakov for their contributions into this project.

\section{References}

1. M. Arnould and K. Takahashi, Rep. Prog. Phys. 62, 395 (1999).

2. A. Mamdouh et al, Nucl. Phys. A679, 337 (2001).

3. J. Dudek et al, Eur. Phys. J. A20, 15 (2004).

4. A. V. Afanasjev and H. Abusara, Phys. Rev. C 78, 014315 (2008).

5. M. G. Itkis et al, Phys. Rev. C 65, 044602 (2002).

6. D. Vretenar et al, Phys. Rep. 409, 101 (2005).

7. A. Sobiczewski and K. Pomorski, Prog. Part. Nucl. Phys. 58, 292 (2007).

8. RIPL-3 stands for reference input parameter library of International Atomic Energy Agency located at http://www-nds.iaea.org/ripl2/.

9. J. Dobrowolski, K. Pomorski, and J. Bartel, Phys. Rev. C75, 024613 (2007).

10. P. Möller et al, Phys. Rev. C79, 064304 (2009).

11. H. Abusara, A. V. Afanasjev, and P. Ring, Phys. Rev. C82, 044303 (2010).

12. J.-P. Delaroche et al, Nucl. Phys. A771, 103 (2006).

13. H. Abusara, A. V. Afanasjev and P. Ring, Phys. Rev. C 85, 024314 (2012).

14. G. A. Lalazissis et al, Phys. Lett. B671, 36 (2009).

15. B.-N. Lu, E.-G. Zhao, and S.-G. Zhou, Phys. Rev. C 85, 011301(R) (2012).

16. V. Prassa et al, Phys. Rev. C 86, 024317 (2012).

17. M. Kortelainen et al, Phys. Rev. C85, 024304 (2012).

18. J. D. McDonnell et al, in preparation.

19. A. V. Afanasjev et al, Int. J. Mod. Phys. E21, 1250025 (2012).

20. M. Kowal et al, Phys. Rev. C82, 014303 (2010).

21. D. Habs, Nucl. Phys. A502, 105c (1989).

22. H. Backe et al, Phys. Rev. Lett. 80, 920 (1998).

23. S. Karatzikos et al, Phys. Lett. B689, 72 (2010).

24. H. C. Britt and J. R. Huizenga, Phys. Rev. C 9, 435 (1974).

25. A. V. Afanasjev and O. Abdurazakov, to be submitted to Phys. Rev. C 